\title{
Tongue necrosis as a complication of vasoconstrictor agents in the intensive care setting
}

\author{
S. Oaleed Noordally $\cdot$ Schoeb Sohawon • \\ Ruth Duttmann • Philippe Gottignies • \\ Jacques Devriendt
}

Received: 20 April 2010/Accepted: 13 May 2010/Published online: 10 June 2010

(c) SIMI 2010

\section{Introduction}

The tongue is well irrigated by the lingual and submandibular arteries along with numerous collaterals that are invariably present. This rich vascular supply makes tongue necrosis exceptional. Tongue necrosis has been reported in giant cell arteritis, Wegener's granulomatosis, malignant tumors, previous radiation to the neck, intraarterial injections, emboli, ergotism, essential thrombocytosis, and calciphylaxis [1-3, 5]. We report a case of tongue necrosis due to a combination of different vasoconstrictive agents in the intensive care setting.

\section{Case report}

A 59-year-old patient was admitted to the emergency department (ED) for hypotension, bradycardia and altered mental status with an APACHE II score of 26. The history revealed a suicide attempt by ingestion of 56 tablets of $10 \mathrm{mg}$ of bisoprolol (Bisoprolol), 30 tablets of $10 \mathrm{mg}$ of amlodipine (Amlor) and 50 tablets of $50 \mathrm{mg}$ of chlorothiazide (Hygroton). His past medical history revealed a

S. O. Noordally $(\varangle) \cdot$ P. Gottignies $\cdot$ J. Devriendt Intensive Care Unit, Department of Critical Care, CHU Brugmann, Free University of Brussels, 4, Place A. Van Gehuchten, 1020 Brussels, Belgium e-mail: Sheikoaleed.Noordally@chu-brugmann.be

\section{S. Sohawon}

Department of Surgery, Erasme Academic Hospital, Free University of Brussels, Brussels, Belgium

\section{R. Duttmann}

Department of Pathology, CHU Brugmann,

Free University of Brussels, Brussels, Belgium history of depression, hypercholesterolemia and essential hypertension. On admission, vital signs were: blood pressure $67 / 45 \mathrm{mmHg}$, pulse rate 40 beats $/ \mathrm{min}$, temperature $35.7^{\circ} \mathrm{C}$, and oxygen saturation $97 \%$ with $5 \mathrm{~L} / \mathrm{min}$ of oxygen. The pulse was full and regular. The patient was sedated and intubated for airway protection. The remaining physical examination was unremarkable on admission.

The patient received fluid resuscitation, atropine and glucagon (Glucagen), and was transferred to the intensive care unit (ICU) where he was started on a glucagon drip $0.2 \mathrm{mg} / \mathrm{h}$ that was weaned within $5 \mathrm{~h}$ of admission. The patient remained hypotensive, cyanotic, and unresponsive to optimal fluid challenges despite central venous pressures oscillating from 14 to $17 \mathrm{mmHg}$ ) with ScVO2 values not below $70 \%$. The heart rate accelerated to 60-65 beats/min and sinus, transvenous pacing was not initiated. Dobutamine was added up to a maximum dose of $20 \mu \mathrm{g} / \mathrm{kg}$ per min. Failure to respond to fluid resuscitation, led to additional continuous infusion of dopamine (Dynatra) at a maximum dose of $20 \mu \mathrm{g} / \mathrm{kg}$ per min, and subsequently norepinephrine (Levophed) was added at a maximum dose of $1 \mu \mathrm{g} / \mathrm{kg}$ per min for $36 \mathrm{~h}$, and thereafter the latter was progressively weaned over $72 \mathrm{~h}$. Due to failure to respond to fluid resuscitation and these vasoactive agents, he was initiated on terlipressin (Glypressin) $1 \mathrm{mg}$ twice daily for 3 days and milrinone (Corotrope) at a maximum dose of $1 \mu \mathrm{g} / \mathrm{kg}$ per min for $24 \mathrm{~h}$, which was weaned over 3 days. Blood tests did not show increased erythrocyte sedimentation rate (ESR) or C-reactive protein (CRP) levels. Arterial blood lactate in the resuscitation periods did not exceed $36 \mathrm{mg} / \mathrm{dL}$, ionized calcium levels did not fall below $1.31 \mathrm{mmol} / \mathrm{l}$, a base excess of not less than -7.1 and urine output of $1.2 \mathrm{ml} / \mathrm{kg}$ per $\mathrm{h}$ for a weight of $55 \mathrm{~kg}$. An electrocardiogram showed a bradycardia at 43 beats/min and sinus and chest radiographs were unremarkable. 
Fig. 1 Necrotic tongue (a), with a hematoxylin and eosin $(\mathrm{H} \& \mathrm{E})$ staining at $\times 100$ magnification showing necrotic striated muscle cells with coagulation necrosis of blood vessels (b)
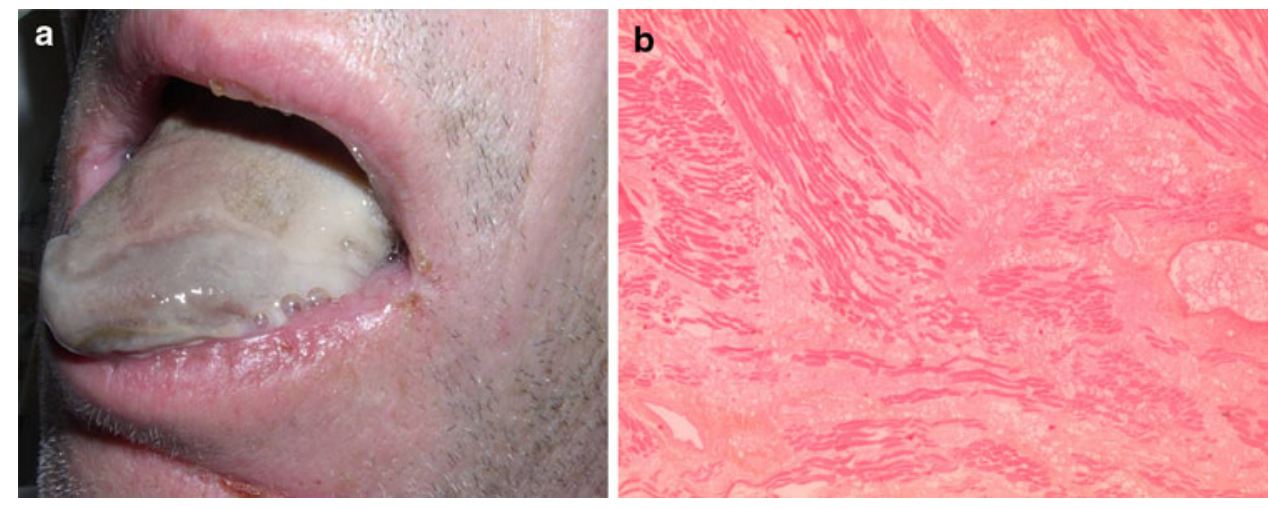

On the fifth day post-admission, peripheral cyanosis persisted including ear lobe cyanosis, scrotal necrosis and marbled skin patterns. On the sixth day, a glossal edema was noted that thereafter became necrotic and blackish in the right anterior quadrant, which was easily sectioned at the bedside. The left anterior quadrant became pearly with loss of sensitivity to touch and temperature (Fig. 1a). The patient underwent extensive tongue debridement and resection. Histologic examination revealed tongue necrosis with absence of nuclei in the muscle fibers and fibrinous material in blood vessels without granulomas (Fig. 1b). The patient recovered gradually and was transferred to our rehabilitation unit, and is recovering well.

\section{Discussion}

The use of vasoconstrictors in the intensive care setting has attendant risks and numerous side effects such as coronary artery constriction, dysrhythmia, bowel ischemia or infarction, ischemia or gangrene of the extremities, cerebral edema and nipple necrosis. We believe that the tongue necrosis in our patient occurred on the basis of vasoconstriction of the lingual and submandibular arteries that supply the tongue because our patient failed to respond to fluid resuscitation. With a history of hypertension and hypercholesterolemia, it is possible that there was underlying atherosclerosis that may have contributed to the ischemia. Among vasoconstrictive agents, intravenous glypressin was used. Glypressin produces constriction of smooth muscle in the walls of arterioles and capillaries. Moreover, glypressin has been recently described as a risk factor for cutaneous necrosis [4]. The ESR levels prior to admission were unknown; however, the ESR levels after ICU stay were regressing towards the normal range with normal CRP values excluding Wegener granulomatosis and Horton's disease. Histopathological analysis revealed ischemic necrosis and intraluminal thrombi without vasculitis (Fig. 1b).

Symptoms of tongue necrosis include pain, glossodynia, lingual claudication, recurrent blanching, erythema and bluish swellings. These symptoms were not identified in our patient; furthermore, the presence of the endotracheal tube may have contributed to the ischemia of the tongue by maintaining a continuous edema, and therefore further reducing the lingual blood flow.

Treatment of tongue necrosis in cases of granulomatous diseases such as Horton's and Wegener's diseases includes steroids with or without nitroglycerine for limited necrosis. Large necrotic areas must be resected under general anesthesia and closed, primarily to avoid recurrent infections [5]. Considerable deformity of the tongue, after fibrosis and shrinkage of the infarcted area, can occur without such management. Our patient had extensive debridement and resection. He recovered gradually, and was recently transferred to a rehabilitation unit, and is recovering well with ongoing speech therapy.

\section{Conclusion}

Tongue necrosis is not a recognized complication of vasoconstrictor use in the intensive care setting. The presented case outlines a rare complication of the use of a combination of powerful vasoconstrictive agents with rapid development of tongue necrosis resulting in excessive tissue loss. This mutilating clinical course underscores the need for prompt recognition, and the necessary measures to be taken in order to limit the extent of tissue loss in the sedated and intubated patient, such as converting the patient to a tracheostomy if persistent high doses of vasoconstrictors remain unavoidable.

Conflict of interest None.

\section{References}

1. Leroy X, Delobelle A, Lefebvre JL, Cabaret V, Bloget F, Vilain MO (1997) Epithelioid sarcoma of the tongue. J Clin Pathol $50: 869-870$ 
2. Fernandez-Casado A, Sanchez-Gonzalez B (2009) Tongue necrosis in a patient with essential thrombocytosis. N Eng J Med 360:28

3. Olesen JB (2009) Necrosis of the tongue triggered by ergotamine in unrecognized temporal arteritis. Ugeskr Laeger 171:125-126

4. Megarbane H, Barete S, Khosrotehrani K et al (2009) Two observations raising questions about risk factors of cutaneous necrosis induced by terlipressin (Glypressin). Dermatology 218:334-337

5. Llorente Pendas S, De Vicente Rodriguez JC, Gonzalez Garcia M, Junquera Guttierrez LM, Lopez Arranz JS (1994) Tongue necrosis as a complication of temporal arteritis. Oral Surg Oral Med Oral Pathol 78:448-451 